\title{
Efectividad en programas preventivos de lesiones deportivas músculo esqueléticas en adultos sedentarios que inician actividad física Effectiveness in preventive programs of skeletal muscle injuries in sedentary adults who begin a sports activity \\ Pablo Vásquez Reyes, Victor Villegas Pulgar, Fernando Romero Carrasco, Cristian José Oñate Navarrete, Claudia Navarrete Hidalgo \\ Universidad Católica de Temuco (Chile)
}

Resumen. El comportamiento sedentario conlleva al aumento de la fatiga muscular, esto debido a que existe menor producción de óxido nítrico, menor flujo sanguíneo, predisponiendo a la generación de lesiones músculos esqueléticas. En Chile la población sedentaria mayor de 18 años alcanza el 86,7\% según la última Encuesta Nacional De Salud 2016-2017. Objetivo: demostrar el efecto de los programas de intervención realizados para prevenir lesiones músculo esqueléticasen personas sedentarias que inician una actividad deportiva. Método: se efectuó una revisión de la literatura científica en bases de datosy en función de criterios de elegibilidad se seleccionaron nueve artículos. Resultados: se escogieron el $18 \%$ de los artículos seleccionados obteniendo nueve artículos de un total de 49 artículos atingentes encontrados en la búsqueda.Conclusiones: se destacan como efectivos aquellos programas con una frecuencia de tres a cinco días; con un volumen de intensidad semanal bajo o medio, según la frecuencia escogida; en los cuales el plan de entrenamiento considera ejercicios de movilidad articular, ejercicios excéntricos y entrenamiento propioceptivo; no se define una duración determinada para la sesión de entrenamiento,para conseguir cambios significativos.

Palabras Claves: Actividad Física, Comportamiento Sedentario, Medicina preventiva, Ejercicio, Lesiones Deportivas.

\begin{abstract}
Sedentary behaviors lead to increased muscle fatigue, this due to lower nitric oxide production, lower blood flow, predisposing to the generation of skeletal muscle injuries. In Chile, sedentary population over the age of 18 years reaches $86.7 \%$ according to the latest National Health Survey 2016-2017.Objective: to demonstrate the effect of intervention programs performed to prevent skeletal muscle injuries. Method: A review of the scientific literature was carried out in databases and nine articles were selected based on eligibility criteria. Results: $18 \%$ of the articles were chosen from a total of 49 in the list of items found in the search. Conclusions: programs with a frequency of three to five days are highlighted as effective; with low or medium weekly intensity volume, it depends on the chosen frequency; the training plans include joint mobility exercises, eccentric exercises, and proprioceptive training; there is no fixed duration for the training session to achieve significant changes.
\end{abstract}

Keywords: Physical Activity, Sedentary Behavior, Preventive medicine, Exercise, Athletic Injuries.

\section{Introducción}

Según Patterson et al. (2018) define el comportamiento sedentario como la realización de cualquier actividad durante la cual una persona está acostada, reclinada o sentada, o actividades que conllevan un bajo gasto energético. Desde el punto de vista energético se describe a una persona sedentaria como aquella que no realiza un gasto energético de al menos 600 Mets*min-1 por semana $(720 \mathrm{kcal}$ por semana en una persona de $70 \mathrm{~kg}$ ), entre actividades vigorosas, moderadas y/o caminata (Seydel et al., 2017). Debido a que el estilo de vida sedentario propio de la vida actual suele englobar una serie de factores de riesgo cardiovasculares, que predisponen a un mayor riesgo de sufrir enfermedades crónicas no transmisibles (ECNT) como la diabetes mellitus, obesidad, síndrome metabólico, entre otras es que la promoción, prescripción y fomento de actividad física se ha hecho fundamental para contrarrestar estas problemáticas (Hutchinson, Prady, Smith, White, Graham, 2015).

Actualmente en Chile existe una gran paradoja que define la relación entre sedentarismo versus la práctica deportiva y de actividad física, donde a pesar de la gran cobertura que realizan los medios de comunicación masivos en torno al incentivo y prácticas de actividad física y deporte la población chilena destaca por ser una de las más sedentarias

Fecha recepción: 25-11-19. Fecha de aceptación: 18-06-20

Cristian José Oñate Navarrete

kinecristianjose@gmail.com de Latinoamérica (MINSAL, 2017). La Encuesta Nacional de Actividad Física y Deporte, aplicada en Chile entre octubre y noviembre del 2018, arrojó resultados que hoy en Chile el 81,3 por ciento de la población adulta se encuentra inactivo físicamente, eso quiere decir que 8 de cada 10 chilenos no realiza actividad física ni deportiva según las recomendaciones de la OMS (Ministerio del Deporte, 2018).

El principal problema radica en que el aumento prolongado del sedentarismo en el tiempo puede llevar a una disminución de la capacidad física a largo plazo afectando el equilibrio, condición cardiovascular, fuerza muscular, flexibilidad, etc. (Patterson et al., 2018). Se han estudiado una serie de modificaciones osteoarticulares asociadas al sedentarismo y su estilo de vida, por ejemplo una revisión sistemática realizada el año 2015 por Crespo-Salgado et al. la cual estudió el efecto de diversas alteraciones metabólicas en la generación de disfunciones tendinosas reconoció el aumento de la adiposidad como factor de riesgo para la generación de tendinopatías asociadas principalmente a alteraciones bioquímicas atribuidas a factores dismetabólicos y a la sobrecarga tendineas (Crespo-Salgado, Delgado-Martin, Blanco-Iglesias, Aldecoa-Landesa, 2015).

Un comportamiento sedentario también conlleva a un aumento de la fatiga muscular puesto que existe menor producción de óxido nítrico, menor vasodilatación y en consecuencia un menor flujo sanguíneo, predisponiendo la generación de lesiones musculoesqueléticas (Pinto et al., 2017)

Desde el contexto nacional uno de los principios más importante que rige la actual política nacional de actividad 
física y deporte para el año 2016 a 2025 es el de:

«Educación, asociatividad y cultura deportiva» este principio recalca que «el estado tiene la responsabilidad de promover el acceso de los ciudadanos a su oferta programática, informando y educando en sus oportunidades, beneficios, valores, y procurando que la cultura deportiva se ejerza de manera activa y en concordancia con el principio de participación social» (Ministerio del Deporte, Gobierno de Chile. 2016, p105).

La presente revisión bibliográfica tiene como objetivo conocer la evidencia científica existente, que permita avalar la efectividad de los programas preventivos de lesiones músculo esqueléticas en adultos sedentarios que desean iniciar una actividad deportiva. Por lo que se debe considerar como punto de partida para la prescripción de estos programas, una evaluación por parte de un médico y el estado de salud actual de los participantes, para luego diseñar una intervención interdisciplinar, a cargo de un Kinesiólogo, para controlar de la mejor manera posible los factores de riesgo presentes en los usuarios (Lurati, 2018).

\section{Material y método}

\section{Estrategia de búsqueda}

La búsqueda de información se realizó mediante una revisión en diferentes bases de datos en el campo de las ciencias de la salud, entre ellas MEDLINE, PubMed, Scopus, Scielo, donde se realizó un chequeo narrativo de la literatura, con límite temporal en la búsqueda de las publicaciones desde el año 2015 hasta el 2020. El método de búsqueda se basó en una estrategia denominada PICOT que considera: Pacientes (P) adultos desde 18 a 65 años, Intervención (I) asociado una búsqueda de programas preventivos de lesiones, medidas de resultados $(\mathrm{O})$ a través de lesiones musculoesqueléticas y por último el Tipo de estudio (T) que fueron revisiones sistemáticas y ensayos clínicos. Conforme a esto se formuló la siguiente pregunta, ¿cuál es la efectividad de los programas preventivos de lesiones musculoesqueléticas en adultos sedentarios que inician una actividad deportiva? cabe mencionar, que en el desarrollo de este trabajo no se tuvo una intervención de comparación (C).

Tabla 1

Resultados búsqueda de Ensayos Clínicos

\begin{tabular}{lll} 
Resultados búsqueda de Ensayos Clinicos & & \\
\hline Nombre de artículo (Norma) & Tipo de estudio / Muestra & Objetivo \\
\hline "High-frequency resistance training does not & Ensayo clínico & Comparar el efecto de \\
promote greater muscular adaptations & $\mathrm{N}: 20$ & tres modalidades de \\
compared to low frequencies in young & C: hombres no entrenados & frecuencias (RT) \\
untrained men" (Barcelo's et al, 2018) & Edad: edad promedio 23 años. & diferentes \\
\hline "Effects of two different injury prevention & Ensayo clínico & Evaluar y comparar los \\
resistance exercise protocols on the & $\mathrm{N}: 32$ personas & efectos de dos \\
hamstring torque-angle relationship: a & C: jugadores recreativos de & programas de \\
randomized controlled trial." (Naclerio et al, fútbol & prevención de lesiones \\
2015 ) & Edad: 22.2 promedio de edad. & diferentes.
\end{tabular}

\begin{tabular}{|c|c|c|c|}
\hline Tipo de estudio / Muestra & Objetivo & Intervención/ filtros de búsqueda & Resultados/Conclusión \\
\hline $\begin{array}{l}\text { Ensayo clínico } \\
\mathrm{N}: 20 \\
\mathrm{C}: \text { hombres no entrenados } \\
\text { Edad: edad promedio } 23 \text { años. }\end{array}$ & $\begin{array}{l}\text { Comparar el efecto de } \\
\text { tres modalidades de } \\
\text { frecuencias (RT) } \\
\text { diferentes }\end{array}$ & $\begin{array}{l}-3 \text { series de } 9-12 \text { reps. } \\
- \text { Protocolos de entrenamiento en máquina } \\
\text { de extensión de rodilla. }\end{array}$ & $\begin{array}{l}\text { Las frecuencias de RT más altas no producen efectos } \\
\text { superiores a las frecuencias más bajas para inducir ganancias } \\
\text { en fuerza muscular e hipertrofia en hombres no entrenados. }\end{array}$ \\
\hline $\begin{array}{l}\text { Ensayo clínico } \\
\mathrm{N}: 32 \text { personas } \\
\mathrm{C}: \text { jugadores recreativos de } \\
\text { fútbol } \\
\text { Edad: } 22.2 \text { promedio de edad. }\end{array}$ & $\begin{array}{l}\text { Evaluar y comparar los } \\
\text { efectos de dos } \\
\text { programas de } \\
\text { prevención de lesiones } \\
\text { diferentes. }\end{array}$ & $\begin{array}{l}\text { RT: } 3 \text { veces por semana por } 6 \text { semanas. } \\
\text {-3 series de } 8 \text { repeticiones. } \\
\text {-Descanso de } 1 \text { minuto entre series. } \\
\text { Ejercicios: } \\
\text { (programa 1) } \\
\text {-Curl nórdico. } \\
\text {-Dead lift single leg. } \\
\text {-Eccentric double stiff-legged dead lift. } \\
\text { (Programa 2) } \\
\text {-single leg squat } \\
\text {-single leg squat variant } \\
\text {-forward lunges on a Bosu. }\end{array}$ & $\begin{array}{l}\text { La combinación de ejercicios de diferente naturaleza } \\
\text { destinada a proteger a los atletas de diferentes tipos de } \\
\text { lesiones podrían ser un método eficaz para evitar lesiones }\end{array}$ \\
\hline $\begin{array}{l}\text { Ensayo clínico } \\
\text { N: } 30 \\
\text { C: corredores sin lesiones de } \\
\text { tobillo o dolor en los últimos } \\
\text { dos meses } \\
\text { Edad: } 18-55\end{array}$ & & $\begin{array}{l}\text { GC: calentamiento, placebo y } \\
\text { elongaciones. } \\
\text { GI: entrenamiento de resistencia y } \\
\text { movilidad de tobillo. }\end{array}$ & $\begin{array}{l}\text { - Tanto la fuerza como el ROM del tobillo y dedos } \\
\text { aumentaron de forma considerable en el grupo de } \\
\text { intervención. } \\
\text {-un plan de prevención de lesiones es efectivo para aumentar } \\
\text { tanto la fuerza, movilidad como la estabilidad de tobillo y } \\
\text { así prevenir lesiones en esta zona. }\end{array}$ \\
\hline Ensayo clínico & $\begin{array}{l}\text { Describir y evaluar un } \\
\text { programa de ejercicios }\end{array}$ & $\begin{array}{l}\text { Ejercicios propuestos: } \\
\text { a) Walking lunges. }\end{array}$ & $\begin{array}{l}\text {-La incidencia de estas lesiones sigue alta, posiblemente al } \\
\text { bajo cumplimiento del programa. }\end{array}$ \\
\hline $\begin{array}{l}\text { N: } 4 \text { clubes aficionados de } \\
\text { fútbol holandés. } \\
\text { Edad: } 18-40 \text { años }\end{array}$ & $\begin{array}{l}\text { pliométricos planteado } \\
\text { para la prevención de } \\
\text { lesiones. }\end{array}$ & $\begin{array}{l}\text { b) Tripling followed by drop lunges. } \\
\text { c) Bounding. } \\
\text {-Duración del programa de } 13 \text { semanas. }\end{array}$ & $\begin{array}{l}\text {-Un programa de ejercicios pliométricos podría fomentar la } \\
\text { adherencia a este y mejorar el rendimiento del salto y carrera, } \\
\text { además de prevenir lesiones en isquiotibiales. }\end{array}$ \\
\hline
\end{tabular}

\begin{tabular}{|c|c|c|c|c|}
\hline $\begin{array}{l}\text { "Effects of a therapeutic foot exercise } \\
\text { program on injury incidence, foot } \\
\text { functionality and biomechanics in long- } \\
\text { distance runners: Feasibility study for a } \\
\text { randomized controlled trial." } \\
\text { (Taddei et al, 2018) }\end{array}$ & $\begin{array}{l}\text { Ensayo clínico } \\
\mathrm{N}: 30 \\
\mathrm{C}: \text { corredores sin lesiones de } \\
\text { tobillo o dolor en los últimos } \\
\text { dos meses } \\
\text { Edad: } 18-55\end{array}$ & & $\begin{array}{l}\text { GC: calentamiento, placebo y } \\
\text { elongaciones. } \\
\text { GI: entrenamiento de resistencia y } \\
\text { movilidad de tobillo. }\end{array}$ & $\begin{array}{l}\text {-Tanto la fuerza como el ROM del tobillo y dedos } \\
\text { aumentaron de forma considerable en el grupo de } \\
\text { intervención. } \\
\text {-un plan de prevención de lesiones es efectivo para aumentar } \\
\text { tanto la fuerza, movilidad como la estabilidad de tobillo y } \\
\text { así prevenir lesiones en esta zona. }\end{array}$ \\
\hline $\begin{array}{l}\text { "The preventive effect of the bounding } \\
\text { exercise programme on hamstring injuries in } \\
\text { amateur soccer players: the design of a } \\
\text { randomized controlled trial." } \\
\text { (Van de Hoeff, et al, 2017) }\end{array}$ & $\begin{array}{l}\text { Ensayo clínico } \\
\mathrm{N}: 4 \text { clubes aficionados de } \\
\text { fútbol holandés. } \\
\text { Edad: } 18-40 \text { años }\end{array}$ & $\begin{array}{l}\text { Describir y evaluar un } \\
\text { programa de ejercicios } \\
\text { pliométricos planteado } \\
\text { para la prevención de } \\
\text { lesiones. }\end{array}$ & $\begin{array}{l}\text { Ejercicios propuestos: } \\
\text { a) Walking lunges. } \\
\text { b) Tripling followed by drop lunges. } \\
\text { c) Bounding. } \\
\text {-Duración del programa de } 13 \text { semanas. }\end{array}$ & $\begin{array}{l}\text {-La incidencia de estas lesiones sigue alta, posiblemente al } \\
\text { bajo cumplimiento del programa. } \\
\text {-Un programa de ejercicios pliométricos podría fomentar la } \\
\text { adherencia a este y mejorar el rendimiento del salto y carrera, } \\
\text { además de prevenir lesiones en isquiotibiales. }\end{array}$ \\
\hline $\begin{array}{lll}\text { RT: } & \text { Frecuencia Semanal } & \text { GC: } \\
\end{array}$ & upo de control $\quad$ GI: $\quad$ G & de intervención & & \\
\hline
\end{tabular}

Tabla 2.

Resultados búsqueda de Revisiones Sistemáticas

\begin{tabular}{|c|c|c|c|c|}
\hline Nombre de artículo & $\begin{array}{l}\text { Tipo de estu } \\
\text { / Muestra }\end{array}$ & Objetivo & Intervención/ filtros de búsqueda & Resultados/Conclusión \\
\hline $\begin{array}{l}\text { "Effects of Resistance Training } \\
\text { Frequency on Measures of Muscle } \\
\text { Hypertrophy: A Systematic Review and } \\
\text { Meta-Analysis" } \\
\text { (Schoenfeld, Ogborn \& Krieger, 2016) }\end{array}$ & $\begin{array}{l}\text { Revisión } \\
\text { sistemática }\end{array}$ & $\begin{array}{l}\text { Determinar los efectos de la } \\
\text { frecuencia de entrenamiento en el } \\
\text { entrenamiento de resistencia sobre } \\
\text { los resultados en medidas } \\
\text { hipertróficas }\end{array}$ & $\begin{array}{l}\text {-Ensayos clínicos publicados en inglés. } \\
1 \text {-Que comparen diferentes frecuencias semanales de } \\
\text { eentrenamiento. } \\
\text {-Que evalúen cambios morfológicos musculares. } \\
\text {-Duración mínima de } 4 \text { semanas. } \\
\text {-Personas sin enfermedad crónica o lesión. }\end{array}$ & $\begin{array}{l}\text {-La optimización de la frecuencia de RT puede } \\
\text { tener implicaciones importantes para maximizar } \\
\text { la hipertrofia muscular. } \\
\text {-No se ha determinado si emplear rutinas } \\
\text { divididas con bajas frecuencias semanales de } \\
\text { entrenamiento por grupo muscular, puede } \\
\text { aumentar la hipertrofia }\end{array}$ \\
\hline $\begin{array}{l}\text { "How many times per week should a } \\
\text { muscle be trained to maximize muscle } \\
\text { hypertrophy? A systematic review and } \\
\text { meta-analysis of studies examining the } \\
\text { effects of resistance training frequency" } \\
\text { (Schoenfeld, Grgich \& Krieger, 2018) }\end{array}$ & $\begin{array}{l}\text { Revisión } \\
\text { sistemática }\end{array}$ & $\begin{array}{l}\text { Realizar una revisión sistemática } \\
\text { y metaanálisis de estudios } \\
\text { experimentales hechos para } \\
\text { investigar los efectos de la } \\
\text { frecuencia semanal al entrenar en } \\
\text { la hipertrofia. }\end{array}$ & $\begin{array}{l}\text {-Ensayos modificados. } \\
\text {-Grupos de entrenamiento aleatorizados. } \\
\text {-Evaluar hipertrofia o cambios en la masa muscular magra. } \\
\text {-Duración mínima de seis semanas. } \\
\text {-Protocolos de entrenamiento para resistencia muscular. }\end{array}$ & $\begin{array}{l}\text { El número de veces que un grupo muscular es } \\
\text { entrenado sobre una base semanal tiene un efecto } \\
\text { significativo sobre los resultados hipertróficos en } \\
\text { base a volumen-equiparado }\end{array}$ \\
\hline $\begin{array}{l}\text { "Effect of Resistance Training Frequency } \\
\text { on Gains in Muscular Strength: A } \\
\text { Systematic Review and Meta-Analysis" } \\
\text { (Grgich, Jozo et al. 2018) }\end{array}$ & $\begin{array}{l}\text { Revisión } \\
\text { sistemática. }\end{array}$ & $\begin{array}{l}\text { Comparar los resultados en la } \\
\text { fuerza muscular con diferentes } \\
\text { frecuencias de entrenamiento }\end{array}$ & $\begin{array}{l}\text {-Estudios en inglés. } \\
\text {-Se comparó diferentes frecuencias de entrenamiento. } \\
\text {-Métodos de evaluación de fuerza muscular (RM), y/o prueba } \\
\text { isocinética } \\
\text {-Duración mínima: } 4 \text { sem. } \\
\text {-Estudio en humanos sin lesión aguda o crónica. }\end{array}$ & $\begin{array}{l}\text {-Mayores frecuencias RT resulta en una mayor } \\
\text { ganancia de fuerza. } \\
\text {-Cuando se equipara volumen, no hubo un efecto } \\
\text { significativo de la frecuencia de RT } \\
\text { sobre las ganancias de fuerza muscular. }\end{array}$ \\
\hline $\begin{array}{l}\text { "The Effect of Weekly Set Volume on } \\
\text { Strength Gain: A Meta-Analysis" } \\
\text { (Ralston et al, 2018) }\end{array}$ & $\begin{array}{l}\text { Revisión } \\
\text { sistemática }\end{array}$ & $\begin{array}{l}\text { Examinar los efectos potenciales } \\
\text { de los diferentes niveles de } \\
\text { volumen semanal }\end{array}$ & $\begin{array}{l}\text {-Duración mínima de } 4 \text { semanas. } \\
\text {-Capacitar al menos } 1 \text { grupo muscular importante } \\
\text {-Varones de } 18-60 \text { años. } \\
\text {-Comparación entre conjuntos simples y múltiples de ejercicios. } \\
\text {-Sujetos de investigación libres de lesiones. } \\
\text {-Evaluación pre y post fuerza muscular }(\mathrm{rm})\end{array}$ & $\begin{array}{l}\text {-Se pueden introducir ganancias en las primeras } \\
\text { etapas del entrenamiento mediante la aplicación } \\
\text { de ejercicios multiarticulares cuando se emplean a } \\
\text { medios y altos volúmenes semanales. }\end{array}$ \\
\hline $\begin{array}{l}\text { "The Effect of Inter-Set Rest Intervals on } \\
\text { Resistance Exercise-Induced Muscle } \\
\text { Hypertrophy" (Henselmann \& } \\
\text { shoedenfeld, 2014) }\end{array}$ & $\begin{array}{l}\text { Revisión } \\
\text { sistemática }\end{array}$ & $\begin{array}{l}\text { Presentar y describir los distintos } \\
\text { efectos fisiológicos generados } \\
\text { acorde a los diferentes periodos } \\
\text { de descanso inter-set encontrados } \\
\text { mediante un proceso sistemático } \\
\text { de búsqueda bibliográfica. }\end{array}$ & $\begin{array}{l}\text {-Estudios que examinen la relación entre los intervalos de } \\
\text { descanso e hipertrofia muscular. } \\
\text {-Estudios publicados en inglés. }\end{array}$ & $\begin{array}{l}\text {-El intervalo de descanso en el entrenamiento, } \\
\text { tiene menos efectos en la hipertrofia que otros } \\
\text { intervalos (ej: volumen). } \\
\text {-El tiempo de descanso para la recuperación, } \\
\text { varía según la intensidad, magnitud de la carga, } \\
\text { sexo, edad, tipo de contracción muscular, orden } \\
\text { de ejercicio y fuerza muscular del individuo. }\end{array}$ \\
\hline
\end{tabular}


Los términos de búsqueda se relacionaron con Actividad Física, Ejercicio, Comportamiento Sedentario, Prevención Primaria, Prevención de Accidentes, Lesiones Deportivas. El operador boleano «AND» $\mathrm{y}$ «OR» se utilizó entre las categorías de búsqueda.

Los títulos de los artículos potencialmente relevantes se recuperaron mediante la estrategia de búsqueda antes mencionada y se eliminaron los duplicados. El 7,1\% $(n=11)$ de un total de 154 de los artículos fueron seleccionados en una primera búsqueda filtrando por revisiones sistemáticas. En una segunda búsqueda se optó por la utilización de un grupo menor de términos de búsqueda (ver tabla 1), se agregó un filtro para ensayo clínicos, dentro de los últimos 5 años de publicación seleccionando un 2,5\% $(n=12)$ de los 468 artículos encontrados. Las referencias relevantes de la literatura publicada fueron incluidas en este estudio cuando cumplieron con los criterios de inclusión.

\section{Criterios de inclusión / exclusión. Tipo de participan-} tes

Se excluyeron estudios con población menor de 18 años o con personas mayores de 65 años, empleando para descartar las palabras claves «children» o «elderly». Además, no se consideraron artículos que estén relacionados a deportistas profesionales o de élite, tampoco fueron seleccionados estudios con adultos que tuviesen discapacidad o con alteraciones psiquiátricas. Sin embargo, es una línea de trabajo futura que podría explorarse para estos grupos poblacionales, pero estaba fuera del alcance de esta revisión de alcance.

\section{Contexto}

El concepto central examinado por esta revisión de alcance está basado en investigar los sistemas de entrenamientos que favorezcan la prevención de lesiones durante la iniciación deportiva en adultos sedentarios basada en el método FITT. Dentro del contexto de revisión se incluyeron estudios con un nivel de evidencia A y B, según la clasificación de Canadian task forse on preventive health care (CTFPHC) explicitado en Manterola, C, \& Zavando, D. (2009) incluyendo en el análisis estudios que incluyeran ensayos clínicos aleatorizados y revisiones sistemáticas de la literatura además se incluyeron referentes bibliográficos de la autora Sandra Mahecha Matsudo (2017), José López Chicharro (2006) y dos manuales de la American College of Sport Medicine(2009 y 2012).

Se excluyeron los estudios que contenían entrenamientos vibratorios, neuromuscular o entrenamientos que estuviesen fuera de los ejes de rehabilitación. No hubo restricción en la ubicación donde se realizaban, por ejemplo, en la escuela o en centros deportivo, o el horario, por ejemplo, durante o después de entrenar.

Los datos fueron extraídos y resumidos a través de lectura crítica usando una tabla Excel para llevar un orden lógico que incluyó el estudio, el país, el tipo de estudio y la muestra (tamaño, edad, sexo), el objetivo del estudio, la intervención realizada (frecuencia, intensidad, tiempo, tipo de ejercicio) las variables estudiadas (duración del ejercicio, uso de implementos, etc.) y los principales resultados del estudio, incluyendo estudios en idioma español e inglés.

\section{Resultados}

Finalmente, de los 622 estudios iniciales identificados a través de las diversas bases de datos, 25 estudios cumplieron con los criterios de inclusión y con un $85 \%$ de acuerdo entre el equipo de investigación según los criterios de elegibilidad (nivel de evidencia, año de publicación, relevancia informativa para el estudio, aplicabilidad de los resultados obtenidos). Se analizaron frecuencia $(n=4)$, intensidad $(n=7)$, tiempo $(\mathrm{n}=0)$ y tipo de ejercicios $(\mathrm{n}=5)$ necesarios para formular un adecuado entrenamiento para toda aquella persona adulta que deseen comenzar a realizar deporte. Los capítulos de libro y/o manuales incluidos $(\mathrm{n}=4)$, artículos científicos anexos $(n=6)$ ambos fueron utilizados para fines de contextualización y/o comparación de resultados. Para organizar la gestión de resultados de la búsqueda se utilizó el software Mendeley para generar la base de datos de análisis y la extracción de estos (ver tabla 2)

\section{Características de los estudios}

Se realizaron estudios en 7 países diferentes (Australia, Brasil, Chile, España, Estados unidos, Suiza y Nueva Zelanda). Donde hubo un total de 587 participantes en los grupos experimentales y 543 participantes en los grupos de control, La edad de todos los participantes osciló entre 31.9 años en promedio. En la tabla y y en la tabla 2 encontrarán los principales resultados y conclusiones de los estudios.

\section{Discusión}

En base al análisis de los diferentes resultados para que un programa de entrenamiento con énfasis en prevención de lesiones sea realmente efectivo debe ser capaz de:

1) Identificar de forma previa el problema de la lesión junto a sus posibles causas y factores de riesgo. Un programa de prevención de lesiones tendrá poco efecto en la población si el programa no está basado en la evidencia (Donaldson, Lloyd, Gabbe, Cook, Young, White \& Finch, 2016). Se puede señalar entonces que el grado de una lesión depende en gran parte al deporte y del tipo de intensidad de la actividad deportiva realizada. Attenborough, Hiller, Smith, Stuelcken, Greene, Sinclair, (2014), encontró que el fútbol, baloncesto y voleibol representan las tasas más altas en recurrencia de lesión de tobillo, destacando principalmente el fútbol como deporte causante del mayor porcentaje de esguinces recurrentes $(61 \%)$ e inestabilidad mecánica de tobillo (38\%). Estos factores a considerar tienen un alto impacto e influencia en el proceso de rehabilitación y reintegración deportiva.

2) Se debe progresar siempre en base a una óptima manipulación de variables, ya que se ha demostrado que mediante una óptima manipulación se puede limitar la meseta de entrenamiento obteniendo mejores resultados en un rango de seguridad, facilitando la capacidad de lograr un mayor nivel de aptitud muscular (ACSM 2009). Por su parte Ekegren, Beck, Climie, Owen, Dunstan, Gabbe (2018) agrega que después de una lesión las personas tienen bajos niveles de actividad física, con elevados niveles de conductas sedentarias. 
3) Respecto a la frecuencia de entrenamiento, Barcelos, Damas, Nóbrega, Ugrinowitsch, Lixandrão, Marcelino Eder Dos Santos \& Libardi, (2018) establecen que una frecuencia de entrenamiento elevada de cinco veces por semana (RT5) no tiene mayores diferencias en los resultados finales en la prevención de lesiones durante la iniciación deportiva en comparación a la aplicación de las otras frecuencias semanales debido a que sus periodos de recuperación más breves entre cada sesión disminuyen la velocidad de progresión en comparación a frecuencias de tres y dos veces a la semana. Estos resultados concuerdan con los de Schoenfeld, Ogborn y Krieger en el año (2016), quienes concluyeron que si bien los resultados analizados favorecieron la tasa de dosis/respuesta a los estudios que avalan frecuencias de entrenamiento más altas, los autores destacan que frecuencias de entrenamiento muy elevadas combinadas con altas intensidades de carga conduce rápidamente a un descenso en el rendimiento por sobre entrenamiento. Por lo tanto, los beneficios que tiene el periodizar la frecuencia de entrenamiento de 2 a 3 veces por semana e incluir periodos regulares de descarga en el transcurso de un ciclo de entrenamiento deben ser priorizados en la elección clínica para evitar así los efectos adversos del sobre entrenamiento durante la ejecución. Esto se reafirma con la frecuencia de entrenamiento recomendada para aquellas personas no entrenadas o que entrenan de forma recreacional entregada por el Colegio Americano de Medicina Deportiva (ACSM) en su publicación «Guidelines for exercise testing and prescription» donde agregan que se debe entrenar cada grupo muscular principal 2-3 veces en la semana con al menos 48 horas de descanso entre cada sesión de entrenamiento para el mismo grupo muscular (American College of Sports Medicine, 2012).

4) Cuando evaluamos los efectos de la frecuencia del entrenamiento semanal sobre las adaptaciones hipertróficas, autores como Schoenfeld, Grgic \& Krieger (2018) señalan que la frecuencia semanal de entrenamiento de resistencia no afecta de manera significativa la hipertrofia muscular siempre y cuando se equipare con el volumen de entrenamiento» (Shoenfeld, Grgic \& Krieger, 2018). Por tanto, para alcanzar un volumen determinado en un menor tiempo, los individuos deberán ajustarlo a la frecuencia semanal recomendadas anteriormente, dividiendo el número de ejercicios en los días dedicados al programa de entrenamiento para alcanzar un volumen óptimo, vinculado a las motivaciones personales. Tavares et al. (2017), demostró que una reducción del entrenamiento de fuerza con una frecuencia de 1-2 veces por semana con una disminución del $50 \%$ de la carga del volumen de entrenamiento previo, es capaz de mantener adaptaciones musculares inducidas de forma previa por el ejercicio en personas sanas no entrenadas. Por el contrario, los resultados demostraron que un cese de la actividad total durante un periodo de 8 semanas posterior a 16 semanas de entrenamiento de fuerza previo realizados a individuos sanos no entrenados se asocia con un descenso considerable en el rendimiento de la fuerza. Estos factores se deben tener en cuenta al momento de cesar cualquier protocolo con énfasis en la prevención de lesiones.

5) Para el volumen de entrenamiento Ralston, Kilgore, Wyatt \& Baker, (2017) concluyó que «se pueden introducir ganancias en las primeras etapas del entrenamiento median- te la aplicación de ejercicios multiarticulares cuando se emplean medios y altos volúmenes semanales» (Ralston et al., 2017), esto es reafirmado por el autor Grgic, Schoenfeld, Davies, Lazinica, Krieger \& Pedisic, (2018) al no encontrar efectos significativos entre la frecuencia de entrenamiento semanal y la ganancia de fuerza cuando se equipara al volumen semanal. Un factor importante a considerar al momento de hacer el cálculo óptimo del volumen semanal adecuado a cada contexto, consiste en utilizar los intervalos de descanso inter-set, así lo demostró un estudio realizado por Henselmann \& Shoenfeld (2014) en el que los sujetos que recibieron intervalos de descanso de un minuto inter-set presentaron una mayor concentración de hormona del crecimiento sérico (posterior a dos horas de haber finalizado el entrenamiento) para el mismo plan de entrenamiento que las personas que fueron sometidas a periodos de descanso de tres minutos. Los autores describen que los resultados obtenidos están asociados principalmente a que «intervalos de descanso limitados no le permiten al cuerpo el tiempo suficiente para restablecer la homeostasis, lo que resulta en una mayor acumulación de estos metabolitos en el músculo que potencian la liberación de hormona del crecimiento» (Henselmann \& Shoenfeld, 2014).

6) Para el criterio de la intensidad del ejercicio, Walhin, J. et al (2018) tuvo por objetivo establecer si el ejercicio diario de intensidad vigorosa, una serie diaria de caminatas en treadmill de intensidad moderada y períodos cortos de caminata durante el día puede evitar cambios negativos en la función metabólica inducida por sobrealimentación a corto plazo y actividad física reducida. Concluyo que si bien la sobre alimentación a corto plazo combinada con la reducción de los niveles de actividad física fue capaz de inducir un estado de resistencia a la insulina, hiperinsulinemia y expresión alterada de varios genes claves del tejido adiposo. La aplicación de las tres modalidades de entrenamiento fue capaz de contrarrestar los efectos inducidos por la falta de actividad física y sobrealimentación en diferentes niveles. Donde fue la adición de carrera de intensidad vigorosa la que registro mejores resultados siendo capaz de evitar los cambios independientes de cualquier efecto neto sobre el desequilibrio energético.

7) Referente a la programación de ejecución de un plan de intervención, los autores Ramskov, Nielsen, Sørensen, Parner, Lind \& Rasmussen, (2016) señalan que una progresión semanal mayor o igual a treinta por ciento, aumenta el riesgo de lesión asociadas a la práctica de running. Por su parte Wu, Wills, Laslett, Oldenburg, Jones \& Winzenberg (2016) al describir asociaciones de actividad física total, tiempo gastado en diferentes intensidades de actividad física y tiempo sedentario con densidad mineral ósea, fuerza muscular de miembro inferior, movilidad y medidas de equilibrio en mujeres de mediana edad demostró que el aumento del tiempo dedicado a la realización de actividad física moderada o vigorosa puede ser más importante que disminuir los tiempos sedentarios o que el aumento del tiempo dedicado a la actividad física ligera para la mantención de la fuerza muscular y el equilibrio. Ravnholt, T et al (2018) por su parte pudo concluir que solo 7 semanas de entrenamiento con sprints de $5 \mathrm{~s}$ seguido de baja y moderada intensidad de carrera fueron eficaces para producir aumentos en la densidad mi- 
neral ósea, así como en los marcadores de recambio óseo plasmáticos. Factor y resultados a tener en cuanto en base futuras nuevas programaciones.

8) A la hora de preguntarnos qué tipo de ejercicio es el óptimo para prevenir lesiones, Naclerio, Larumbe-Zabala, Monajati \& Goss-Sampson, (2015) evaluaron y compararon los efectos de dos programas de prevención de lesiones en tres grupos aleatorios (a) un programa de prevención de lesiones para isquiotibial con ejercicios de base de tipo excéntrico, (b) un programa de prevención de lesiones para isquiotibial con ejercicios de base con plataforma inestable y (c) un grupo de control que no fue sometido a ningún plan de prevención. Concluyendo que la combinación de ejercicios de diferente naturaleza destinada a proteger a los atletas de diferentes tipos de lesiones podrían ser un método eficaz para evitarlas, no así en el grupo control. Taddei, Matias, Ribeiro, Inoue, Bus \& Sacco (2018) investigaron los efectos de un programa de ejercicios terapéuticos en la prevención de lesiones en corredores recreativos de larga distancia demostrando que un plan de prevención de lesiones es efectivo para aumentar el ROM del tobillo y dedos del pie, previniendo así lesiones en esta zona. Solo un estudio de los encontrados demostró resultados negativos frente a la aplicación de un programa de prevención de lesiones, este fue realizado por Van de Hoef, Huisstede, Brink, De Vries, Goedhart \& Backx (2017) en un plan de prevención de lesiones para isquiotibiales basado en un programa de ejercicios (Ejercicios de una pierna de salto, con ciclo de estiramientoacortamiento). Tras la intervención se encontró que la incidencia de este tipo de lesiones todavía era alta, posiblemente debido al mal cumplimiento de este programa preventivo de lesiones y quizás esto podría cambiar si se realizaran ejercicios de carácter pliométrico, por su fácil ejecución y poco uso de espacio e implementación deportiva, además de poder mejorar el sprint y salto en estos deportistas a largo plazo. Por su parte Borghuis, Hof y Lemmick (2008) reafirmaron a través de su estudio: «The Importance of SensoryMotor Control in Providing Core Stability Implications for Measurement and Training» que «un deficiente control propioceptivo del CORE puede contribuir a una disminución del control neuromuscular del miembro inferior» (Borghuis, Hof y Lemmick, 2008) favoreciendo el desarrollo de lesiones musculoesqueléticas en el miembro inferior. Resaltando la importancia del trabajo del CORE en la prevención de lesiones. Respaldando por los resultados obtenidos por León, Soto, Araneda, Venegas \& de la Rosa (2019).

9) En cuanto a las modalidades de ejercicio un estudio realizado por Campos, Víllora Gómez \& Martins (2019) que tenía por objetivo analizar los beneficios de diferentes programas de entrenamiento en parámetros (peso, masa grasa, masa magra, fuerza máxima y fuerza explosiva). Demostró que el entrenamiento de fuerza fue quien obtuvo mayores resultados en torno a la fuerza máxima y explosiva, en cuanto a la pérdida de peso y la masa grasa los grupos que realizaron boxeo y entrenamiento de potencia de alta intensidad fueron quienes obtuvieron mayor resultado (Campos et al., 2019).

10) En cuanto a la ausencia de resultados encontrados en torno a la variable de tiempo por sesión, podríamos decir que a pesar de que la literatura dice que «Para adultos, la mayoría de los beneficios de salud se concretan haciendo actividad física, 2 horas y 30 minutos a la semana a una intensidad moderada» (Mahecha, 2017), agregando que no es recomendable que este tiempo se debe realizar en una sola sesión, sino dividirlo. Además, López Chicharro recomienda, que para el entrenamiento de fuerza debe haber 48 horas de descanso entre sesiones. LAACSM agrega, además, que «programar sesiones con una duración mayor a 60 minutos está asociado con altos porcentajes de abandono de la práctica de actividad física». Como grupo consideramos que lo propuesto anteriormente pierde importancia tanto al programar como ejecutar programas de prevención de lesiones del miembro inferior, ya que, según los resultados aportados por la investigación realizada, encontramos que, en todos los programas de prevención de lesiones encontrados, se despreciaba la variable de tiempo por sesión, dándole más énfasis a la importancia del volumen semanal adecuada para cada grupo de intervención.

\section{Conclusiones}

Diferentes estudios indican la importancia de la comprensión específica del contexto de los deportistas (donde entrenan, su deporte, plan de entrenamiento, edad, nivel competitivo, creencias, entre otras), para poder realizar una óptima intervención, la cual debe incluir ejercicios de carácter progresivo e individualizados para cada uno de los deportistas (Lamotte, 2016). Sin embargo, no existe información sobre una individualización del trabajo, sólo se analiza el trabajo desde una perspectiva grupal, enfocado en el deporte a practicar. Una de las limitaciones de este estudio es que no se analizaron los tipos de entrenamiento con variables tales como la alimentación, horas de sueño, días de descanso, características psicológicas (motivaciones deportivas, situación emocional, presencia de estrés), rol social (si trabajan, en que trabajan), equipamiento y protección del atleta (tipo de calzado que ocupa), medio ambiente donde se desempeña el deportista (pasto o cemento), factores claves para poder entender de mejor manera los factores influyentes que pueden jugar un rol importante en la presencia o no de lesiones durante los entrenamientos, tal como señala Hurtado, Santos \& Torres (2015).

Se observa que la mayoría de los estudios no consideran antecedentes mórbidos en las investigaciones, como enfermedades crónicas (HTA, diabetes, osteoporosis, artrosis, entre otras). ya que sólo participaron adultos sedentarios o deportistas amateurs que no presentaban lesiones previas asociadas con la investigación (a excepción de una sola investigación que tomó como grupo control a deportistas que presentaban inestabilidad crónica de tobillo) por tanto sería importante poder evaluar estudios que analizaran con una perspectiva asociada a dichos factores.

También, es importante mencionar que aquellos estudios que hablaban de un plan de prevención para deportistas amateur consideraban un grupo de estudio muy reducido $(\mathrm{n}<50)$, para poder extrapolar sus resultados a todos aquellos deportistas que practican estos deportes. además, estos artículos se basaban solo en cuatro deportes como son fútbol, básquetbol, voleibol y atletismo (este último sólo aquellos que eran velocistas y fondistas), por lo que sus resulta- 
dos igualmente deberían relacionarse con otros deportes masivos, tanto de trabajo en equipo como individuales, como lo son el tenis o el ciclismo (también deportes de contacto, como la lucha o el rugby). Asimismo, no todas las intervenciones contaban con un seguimiento a largo plazo para corroborar que estos planes de entrenamiento puedan significar una disminución en las lesiones asociadas a miembros inferiores.

Faltan investigaciones que evalúen la efectividad de la realización de programas de prevención de lesiones en personas sedentarias debido a que aquellas que han sido realizadas, toman en cuenta un grupo muy acotado de actividades deportivas. Si bien Guex, Wicht, Besson, Degache, Gojanovic \& Gremion, (2020) en su investigación analiza un programa de entrenamiento a largo plazo ( 38 meses), sus resultados no pueden ser generalizados ya que solo se consideró un solo participante en la evaluación. Por lo tanto se requiere una comprensión más profunda del tema respecto a las respuesta del entrenamiento físico y las diversas variables que influyen en la prevención de lesiones en el largo plazo.

\section{Referencias}

American College of Sports Medicine. (2012). ACSM's resource manual for guidelines for exercise testing and prescription. Lippincott Williams \& Wilkins.

American College of Sports Medicine. (2009). Progression Models in Resistance Training for Healthy Adults. Medicine \& Science in Sports \& Exercise, 41(3), 687-708. doi:10.1249/mss.0b013e3181915670

Barcelos, C., Damas, F., Nóbrega, S. R., Ugrinowitsch, C., Lixandrão, M. E., Marcelino Eder Dos Santos, L., \& Libardi, C.A. (2018). High-frequency resistance training does not promote greater muscular adaptations compared to low frequencies in young untrained men. European Journal of Sport Science, 18(8), 1077-1082. doi:10.1080/ 17461391.2018.1476590

Borghuis, J., Hof, A. L., \& Lemmink, K. A. (2008). The importance of sensory-motor control in providing core stability. Sports medicine, 38(11), 893-916.

Campos, F., Víllora, S. G., Gómez, D. G., \& Martins, F. M. L. (2019). Benefits of 8-week fitness programs on health and fitness parameters. Retos: nuevas tendencias en educación física, deporte y recreación, (35), 224-228.

Crespo-Salgado, J. J., Delgado-Martín, J. L., Blanco-Iglesias, O., \& Aldecoa-Landesa, S. (2015). Guía básica de detección del sedentarismo y recomendaciones de actividad física en atención primaria. Atención primaria, 47(3), 175 183.

DiStefano, L. J., Marshall, S. W., Padua, D. A., Peck, K. Y., Beutler, A. I., de la Motte, S. J., ... \& Cameron, K. L. (2016). The effects of an injury prevention program on landing biomechanics over time. The American journal of sports medicine, 44(3), 767-776.

Donaldson, A., Lloyd, D. G., Gabbe, B. J., Cook, J., Young, W., White, P., \& Finch, C. F. (2016). Scientific evidence is just the starting point: a generalizable process for developing sports injury prevention interventions. Journal of sport and health science, 5(3), 334-341.
Ekegren, C. L., Beck, B., Climie, R. E., Owen, N., Dunstan, D. W., \& Gabbe, B. J. (2018). Physical activity and sedentary behavior subsequent to serious orthopedic injury: A systematic review. Archives of physical medicine and rehabilitation, 99(1), 164-177.

Grgic, J., Schoenfeld, B. J., Davies, T. B., Lazinica, B., Krieger, J. W., \& Pedisic, Z. (2018). Effect of resistance training frequency on gains in muscular strength: a systematic review and meta-analysis. Sports Medicine, 48(5), 12071220.

Guex, K., Wicht, S., Besson, C., Degache, F., Gojanovic, B., \& Gremion, G. (2020). From Sedentary and Physical Inactive Behaviours to an Ultra Cycling Race: A Mixed-Method Case Report. International Journal of Environmental Research and Public Health, 17(2), 502.

Henselmans, M., \& Schoenfeld, B. J. (2014). The effect of inter-set rest intervals on resistance exercise-induced muscle hypertrophy. Sports Medicine, 44(12), 1635-1643.

Hurtado J., Santos J., Torres I. (2015). Factores de riesgo intrínsecos funcionales de lesiones deportivas en dos selecciones universitarias de voleibol de la ciudad de Cali. 12 de diciembre del 2018. http:// bibliotecadigital.univalle.edu.co/bitstream/10893/10281/ 1/CB-0566462.pdf

Hutchinson, J., Prady, S., Smith, M., White, P., \& Graham, H. (2015). A scoping review of observational studies examining relationships between environmental behaviors and health behaviors. International journal of environmental research and public health, 12(5), 48334858.

Lamotte, M. (2016). Factores de riesgo cardiovascular y actividad física. EMC-Kinesiterapia-Medicina física, 37(2), 1-7.

León, A. F., Soto, V., Araneda, O., Venegas, R. G., \& de la Rosa, F. J. B. (2019). Muscle activity of the Lumbo-pelvichip complex in three isometric exercises using TRX ${ }^{\circledR}$ rip trainer ${ }^{\mathrm{TM}}$. Retos: nuevas tendencias en educación física, deporte y recreación, (35), 216-220.

López Chicharro, J., \& Fernández, A. (2006). Fisiología del Ejercicio. $3^{\circ}$ edición Madrid: Panamericana.

Lurati,A. R. (2018). Health Issues and Injury Risks Associated with Prolonged Sitting and Sedentary Lifestyles. Workplace health \& safety, 66(6), 285-290.

Mahecha, S., (2017). Actividad física y ejercicio en salud y enfermedad, Santiago, Chile: editorial mediterráneo.

Ministerio del Deporte, Chile. Resumen ejecutivo Encuesta Nacional de Actividad Física y Deporte 2018 en Población de 18 años y más [Internet]. Santiago: Ministerio del Deporte; 2018 Dic [citado 20 Jun 2019]. Disponible en: http://www.mindep. cl/encuesta-actividad-fisica-y-deporte-2018.

Orellana K. et al. (2016). «Política Nacional de Actividad Física y Deporte 2016-2025». MINDEP. Extraído el 01 de abril del 2019 de: http://www.mindep.cl/wp-content/uploads/ 2015/05/POLITICA-ULTIMA-VERSI\%C3\%93N021116.pdf

Naclerio, F., Larumbe-Zabala, E., Monajati, A., \& GossSampson, M. (2015). Effects of two different injury prevention resistance exercise protocols on the hamstring torque-angle relationship: a randomized controlled trial. 
Research in Sports Medicine, 23(4), 379-393. doi:10.1080/ 15438627.2015.1076418 https://www.ncbi.nlm.nih.gov/ pubmed/26264751

Manterola D, Carlos, \& Zavando M, Daniela. (2009). Cómo interpretar los «Niveles de Evidencia» en los diferentes escenarios clínicos. Revista chilena de cirugía, 61(6), 582595. https://dx.doi.org/10.4067/S0718-40262009000600017

Patterson, R., McNamara, E., Tainio, M., de Sá, T. H., Smith, A. D., Sharp, S. J., ... \& Wijndaele, K. (2018). Sedentary behaviour and risk of all-cause, cardiovascular and cancer mortality, and incident type 2 diabetes: a systematic review and dose response meta-analysis.

Pinto, A. J., Roschel, H., de Sá Pinto, A. L., Lima, F. R., Pereira, R. M. R., Silva, C. A., ... \& Gualano, B. (2017). Physical inactivity and sedentary behavior: Overlooked risk factors in autoimmune rheumatic diseases? Autoimmunity reviews, 16(7), 667-674.

Ralston, G. W., Kilgore, L., Wyatt, F. B., \& Baker, J. S. (2017). The effect of weekly set volume on strength gain: a metaanalysis. Sports Medicine, 47(12), 2585-2601.

Ramskov, D., Nielsen, R. O., Sørensen, H., Parner, E., Lind, M., \& Rasmussen, S. (2016). The design of the run Clever randomized trial: running volume,, intensity and runningrelated injuries. BMC musculoskeletal disorders, 17(1), 177.

Seydel, G. S., Kucukoglu, O., Altinbas,A., Demir, O. O., Yilmaz, S., Akkiz, H., ... \& Canbay, A. (2017). Economic growth leads to increase of obesity and associated hepatocellular carcinoma in developing countries. Annals of hepatology, 15(5), 662-672.

Subsecretaría de Salud Pública. MINSAL. [Online].; 2017. Disponible en: https:/www.minsal.cl/wp-content/ uploads/2017/11/ENS-2016-17_PRIMEROSESULTADOS.pdf
Schoenfeld, B. J., Ogborn, D., \& Krieger, J. W. (2016). Effects of resistance training frequency on measures of muscle hypertrophy: a systematic review and meta-analysis. Sports Medicine, 46(11), 1689-1697.

Schoenfeld, B. J., Grgic, J., \& Krieger, J. (2019). How many times per week should a muscle be trained to maximize muscle hypertrophy? A systematic review and metaanalysis of studies examining the effects of resistance training frequency. Journal of sports sciences, 37(11), 1286-1295.

Taddei, U. T., Matias, A. B., Ribeiro, F. I. A., Inoue, R. S., Bus, S.A., \& Sacco, I. C. N. (2018). Effects of a therapeutic foot exercise program on injury incidence, foot functionality and biomechanics in long-distance runners: Feasibility study for a randomized controlled trial. Physical Therapy in Sport. doi:10.1016/j.ptsp.2018.10.015

Tavares, L. D., de Souza, E. O., Ugrinowitsch, C., Laurentino, G. C., Roschel, H., Aihara, A. Y., ... \& Tricoli, V. (2017). Effects of different strength training frequencies during reduced training period on strength and muscle crosssectional area. European journal of sport science, 17(6), 665-672.

Van de Hoef, S., Huisstede, B. M. A., Brink, M. S., De Vries, N., Goedhart, E.A., \& Backx, F. J. G. (2017). The preventive effect of the bounding exercise programme on hamstring injuries in amateur soccer players: the design of a randomized controlled trial. BMC musculoskeletal disorders, 18(1), 355.

Wu, F., Wills, K., Laslett, L. L., Oldenburg, B., Jones, G., \& Winzenberg, T. (2017). Moderate to vigorous physical activity but not sedentary time is associated with musculoskeletal health outcomes in a cohort of Australian middle aged women. Journal of Bone and Mineral Research, 32(4), 708-715.

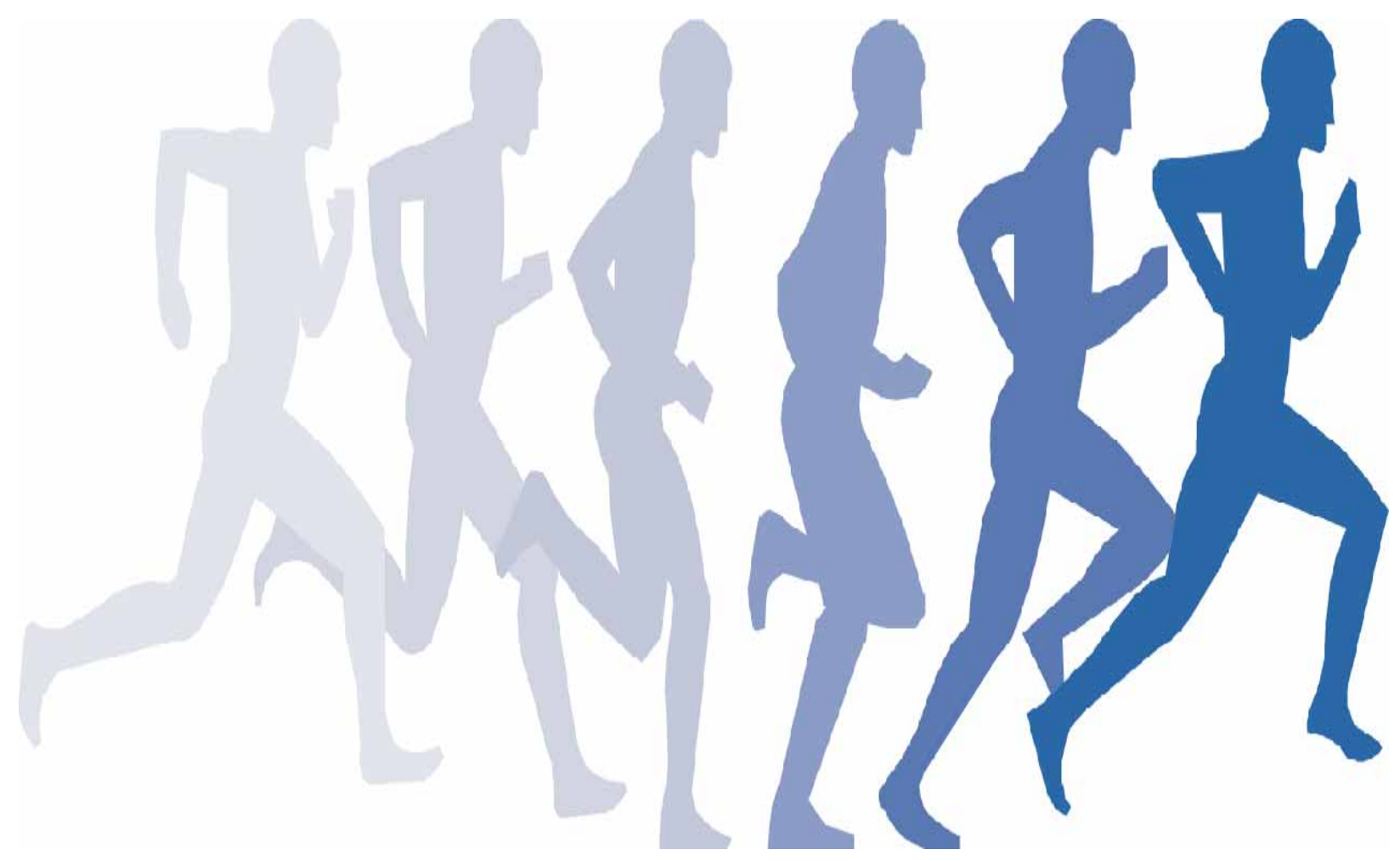

